\title{
O Impacto do Coronavírus (COVID-19) as atividades odontológicas: desafios
}

\section{econômicos e mentais}

\author{
The impact of coronavirus (COVID-19) on dental activities: economic and mental challenges \\ El impacto del coronavirus (COVID-19) en las actividades dentales: desafíos económicos y mentales
}

Recebido: 15/12/2020 | Revisado: 16/12/2020 | Aceito: 07/01/2021 | Publicado: 09/01/2021

Pamela Gomes

ORCID: https://orcid.org/0000-0002-7545-5056 Hospital Regional de Tucuruí, Brasil

E-mail: pamelacassia@ hotmail.com

Wellington Vieira

ORCID: https://orcid.org/0000-0002-6701-324X Associação Paulista de Cirurgiões Dentistas-Santo André, Brasil

E-mail: vieira_well@hotmail.com

Rudiney Daruge

ORCID: https://orcid.org/0000-0002-5735-5756 Centro de Estudo e Pesquisas Prof. Dr. Walter K. Daruge, Brasil E-mail: rudiney@ceddar.org.br

Christian Recchioni

ORCID: https://orcid.org/0000-0003-0967-4530 Universidade Nilton Lins, Brasil

E-mail: christianrecchioni@yahoo.com.br

Camila Pugliese

ORCID: https://orcid.org/0000-0002-7609-9161 Faculdade São Leopoldo Mandic, Brasil E-mail: cami2_pugliese@hotmail.com

Rayssa Villafort

ORCID: https://orcid.org/0000-0003-4575-6218

Complexo Hospitalar São Francisco, Brasil E-mail: rayssavillafort01@hotmail.com

Wilson Cirilo

ORCID: https://orcid.org/0000-0002-7457-3448

Santa Casa de Misericórdia de Quintana, Brasil E-mail: rayssavillafort01@hotmail.com

Kelly Zaia Barel

ORCID: https://orcid.org/0000-0001-7782-329X Faculdade São Leopoldo Mandic, Brasil E-mail: kellybarel@yahoo.com.br

\begin{abstract}
Resumo
A infecção pelo novo Coronavírus 2019 (COVID-19) tem causado um grave impacto na saúde mundial, nos setores hospitalar e odontológico. O Brasil registrou o segundo maior número de pacientes e mais óbitos pelo coronavírus no mundo, perdendo apenas para os Estados Unidos. Os meios de comunicação social e científicas afirmaram que cirurgiões dentistas foram considerados grupo de risco para o covid-19, podendo atuar como vetor de transmissão direta e indireta em sua rotina de atendimento odontológico. Diante do ocorrido novas regras e hábitos amparados pela Organização Mundial de Saúde, alertaram sobre os atendimentos e procedimentos Odontológicos fossem realizados em caso de necessidade extrema; ou seja; apenas atendimentos de urgência e emergência, e casos eletivos fossem postergados, como medida de prevenção da disseminação do COVID-19, o distanciamento social adotado no Brasil pode ter consequências econômicas e psicossociais para a odontologia. Neste contexto, o presente estudo, os autores realizaram uma pesquisa nacional com cirurgiões dentistas brasileiros para avaliar o impacto da pandemia do novo Coronavírus sobre as atividades odontológicas.Um total de 246 cirurgiões dentistas responderam à pesquisa e os resultados indicaram que a grande maioria dos profissionais tiveram renda diminuída no período, seja pelos cancelamentos de consultas ou diminuição o número de atendimentos. Eles têm consciência que estão mais expostos, e por este motivo realizaram investimento científico e aquisição de equipamentos de proteção individual e essas junções de fatores podem provocar maior prejuízo no período de pandemia.
\end{abstract}

Palavras-chave: COVID-19; Cirurgião-dentista; Impacto-econômico; Coronavírus-19; Pandemia.

\section{Abstract}

Infection with the new Coronavirus 2019 (COVID-19) has had a serious impact on global health in the hospital and dental sectors. Brazil recorded the second highest number of patients and more deaths from coronavirus in the world, 
second only to the United States. The social and scientific media stated that dental surgeons were considered a risk group for COVID-19, and could act as a vector for direct and indirect transmission in their dental care routine. In view of the occurrence of new rules and habits supported by the World Health Organization, they warned about dental care and procedures to be carried out in case of extreme need; that is; only urgent and emergency care, and elective cases were postponed, as a measure to prevent the spread of COVID-19, the social distance adopted in Brazil can have economic and psychosocial consequences for dentistry. In this context, the present study, the authors conducted a national survey with Brazilian dentists to assess the impact of the New Coronavirus pandemic on dental activities. A total of 246 dentist surgeons responded to the survey and the results indicated that the vast majority of professionals had decreased income in the period, either by canceling appointments or decreasing the number of visits. They are aware that they are more exposed, and for this reason they made scientific investments and purchased individual protection equipment, and these combinations of factors can cause greater damage in the pandemic period.

Keywords: COVID-19; Dental surgeon; Economic-impact; Coronavirus-19; Pandemic.

\section{Resumen}

La infección por el nuevo virus Corona 2019 (COVID-19) ha tenido un impacto grave en la salud mundial en los sectores hospitalario y dental. Brasil registró el segundo mayor número de pacientes y más muertes por coronavirus en el mundo, solo superado por Estados Unidos. Según la información científica y de los medios de comunicación, afirmaron que los cirujanos dentales eran considerados un grupo de riesgo para el COVID-19 y podían actuar como vector de transmisión directa e indirecta en su rutina de atención dental. Ante la ocurrencia de nuevas normas y hábitos apoyados por la Organización Mundial de la Salud, alertaron sobre los cuidados y procedimientos dentales a realizar en caso de extrema necesidad; o sea; Sólo se pospuso la atención de urgencia y emergencia, y los casos electivos, como medida para prevenir la propagación del COVID-19, la distancia social adoptada en Brasil puede tener consecuencias económicas y psicosociales para la odontología. En este contexto, el presente estudio, los autores realizaron una encuesta nacional con dentistas brasileños para evaluar el impacto de la pandemia del Nuevo Coronavirus en las actividades dentales. Un total de 246 cirujanos dentistas respondieron a la encuesta y los resultados indicaron que la gran mayoría de los profesionales había disminuido sus ingresos en el período, ya sea por cancelaciones de citas o por disminución en el número de visitas, son conscientes de que están más expuestos, y por ello Realizó inversión científica y compra de equipo de protección personal y estas combinaciones de factores que pueden causar mayores daños en el período pandémico.

Palabras clave: COVID-19; Cirujano dentista; Impacto económico; Coronavirus-19; Pandemia.

\section{Introdução}

A Organização Mundial de Saúde (OMS), confirmou na china, o surto da COVID-19 em dezembro de 2019, causada pelo vírus de cepa Coronavírus. A COVID-19 foi considerada uma pandemia em função do seu poder de alcance, ocasionando grande números de adoecimento relacionados as vias respiratórias e óbitos mundiais, exigindo uma ação epidemiológica para combate e controle aos problemas ocasionados pela SARS-CoV-2 (WHO,2020). A sintomatologia característica do COVID-19 são: febre, fadiga, tosse seca, associadas à mialgia e dispneia (MS, 2020; Peng et al., 2020).

No último relatório do Ministério da Saúde, publicado em 14 de dezembro de 2020, estão documentados 6.901 .952 casos confirmados, 181.402mortes, com registro em países de todas as regiões do mundo3. No Brasil, a primeira notificação do COVID 19 ocorreu em 25 de fevereiro de 20204 e desde então o número de acometidos pelo vírus tem crescido, gradativamente. O ministério da saúde divulgou em seu site uma síntese dos casos ocorridos nas regiões brasileiras: sudeste com 2.409.036, seguidos da região nordeste 1.751.622, sul 1.143.101, centro-oeste com 815.043 e o Norte com 808.343 (MS,2020).

No que se refere aos aspectos epidemiológicos aos profissionais de saúde, o ministério da saúde lançou uma cartilha a fim de mantê-los informados quanto as questões de transmissão, e prevenção mediante a determinados procedimentos odontológicos eficazes em relação a COVID-19. Uma das principais informações foram sobre as vias de transmissão diretas são: tosse, espirro, inalação de gotículas ou transmissão por contato com a via oral, nasais ou membranas mucosas dos olhos, e por superfícies contaminadas (WHO,2020; Banerjee,2020). Os motores odontológicos são os principais são as principais rotas diretas de transmissão: os aerossóis, priorizando-os como grupos de risco para a infecção do COVID-19 .Justificando a argumentação aos odontólogos á priorizassem os atendimentos emergenciais de seus pacientes, seguidos previamente de bochechos com clorexidina $0,12 \%$ ou água oxigenada 10 volumes (WHO,2020; Banerjee,2020).Os cirurgiões dentistas são os 
profissionais aptos a identificar, solicitar exames: laboratoriais e exames de imagens como a tomografia, necessários em casos suspeitos de COVID-19, relacionadas aos sinais, sintomas, grupos de risco e gravidade da doença (Mores et al., 2009).

Estudos demonstram que a China foi o primeiro país que adotou a quarentena, o isolamento social como medidas protetivas a disseminação do novo Coronavírus indicam que há consequências psicológicas em massa, tais como ansiedade, depressão, uso excessivo de álcool, solidão, e menor bem estar mental em relação aos índices populacionais usuais (Ahmed et al.,2020).

Associado a isso, pode constatar perdas econômicas nos locais mais afetados e crescente preocupação no setor econômico em consequência da pandemia do COVID-196, o risco psicossocial está intimamente interligado a recessão econômica, pobreza e desemprego (Przybylski et al., 2020), mediante essas hipóteses os autores questionaram de que forma a pandemia poderia afetar negativamente a saúde mental e financeira dos odontólogos durante o episódio pandêmico.

\section{Metodologia}

No presente estudo, os autores realizaram uma pesquisa, com cirurgiões dentistas brasileiros com intuito de avaliar qual foi o impacto do Coronavírus nas rotinas odontológicas desses profissionais sobre: o status de conhecimento pandêmico do COVID-19, as características epidemiológicas dos profissionais: gênero, idade, grupo de risco, a relação das regiões brasileiras, atendimento clínico: tipos de procedimentos realizados, as especialidades, alterações laboratoriais, prevenção, sintomas sugestivos, diretrizes de tratamento e o fator econômico.

A metodologia utilizada foi um estudo de caso descritivo, qualitativo, quantitativo, exploratório e inferencial, tendo como cenário a pandemia do COVID-19 nas rotinas odontológicas no Brasil. O estudo de caso na área da saúde tem a finalidade de investigar analisar e descrever um fenômeno de forma a validar uma pesquisa científica (Pereira et al., 2019). O Estudo de caso pode ser considerado complexo e completo; pois descreve, analisa e observa dados: qualitativos e quantitativos. Segundo Yin (2015) dados qualitativos e qualitativos são complementares em um amplo estudo investigativo.

Esse projeto de pesquisa teve a participação de 246 cirurgiões dentistas de ambos os sexos, de todas as regiões brasileiras, e que aceitaram participar do estudo, seguindo os preceitos éticos do estudo, assinando o Termo de Consentimento Livre e Esclarecido (TCLE).O mesmo foi aprovado pelo Comitê de Ética em Pesquisa/CEP da Faculdade de Medicina e Odontologia da São Leopoldo Mandic, com parecer de aprovação nº 4.359.650.

A coleta de dados desse projeto foi realizada pelos mestrandos de cirurgia e Traumatologia Bucomaxilofacial utilizando os seguintes instrumentos: Questionário sociodemográfico econômico; Questionário de Condições de Saúde; Questionário sobre o conhecimento da Pandemia do COVID-19, Questionário sobre aquisição de EPIS. Todos os instrumentos foram implantados para o banco de informações pelos mestrandos de cirurgia, através da plataforma de questionários online Google Forms. O instrumento de coleta de dados utilizado foi um questionário on line, composto de 21 questões ordenadas de perguntas fechadas e abertas, respondidos sem a presença do entrevistador (Marconi \& Lakatos, 2003). O questionário online utilizado neste período de quarentena ou isolamento social, possibilitou alcançar grande número de participantes em anonimato, além de não sofrer influência de opiniões do entrevistador, o questionário virtual, consiste em questões fechadas e uma questão aberta. A técnica de amostragem utilizada foi a amostra por conveniência devido ao período curto de permanência da pesquisa: 16 de agosto a 16 de setembro de 2020 (30 dias corridos), levando em consideração a disponibilidade de pessoas neste intervalo de tempo, resultando em um método mais prático, fácil, objetivo em seus resultados.

A amostra de conveniência do nosso projeto consta com uma parte dos cirurgiões dentistas que aderiram ao questionário neste cenário de Pandemia, permitindo observar e coletar informações críticas que devem ser resolvidas imediatamente.

Os critérios de inclusão foram: ser cirurgião dentista com atuação nas regiões brasileiras.Com a finalidade de garantir 
maior fidelidade nas informações e por não ser objeto de investigação, excluíram-se os demais profissionais.

A análise de dados foi utilizada pela plataforma Google Forms, separados e organizados em gráficos, quadros e tabelas, sendo os resultados apresentados em relação a quantidade e tipo de respostas de cada indivíduo, separados em categorias definidas pelos próprios questionários, finalizados de maneira rápida e segura.

\section{Resultados e Discussões}

A pesquisa em questão obteve adesão de 246 cirurgiões dentistas de todos os estados brasileiros responderam à pesquisa (Figura 1). Os profissionais com maior adesão ao nosso questionário são do gênero: feminino (61\%), com maior prevalência entre 20 a 30 anos (31,3\%), seguido pelos profissionais acima de 20 anos de formado (32,9\%), com 24,9\% dos CDS consideram-se grupo de risco. Diante dos dados obtidos, 210 cirurgiões dentistas foram unânimes em relatar que a rotina dos atendimentos odontológicos sofreu um impacto de $85,4 \%$ na queda do faturamento, com predominância na região sudeste $76,9 \%$, seguidos da região Norte $8,3 \%$, região Sul 4,1\%,e empate entre as regiões Nordeste e Distrito Federal com 3,7\% e região Centro-Oeste com 3,3\% de cirurgiões dentistas atuantes nas regiões Brasileiras (Figura 2), ou seja, ainda que a pandemia tenha ocasionado um cenário de crise econômica brasileira, percebe -se as disparidades econômicas distribuídas ao longo do estudo.

Figura 1 - Distribuição epidemiológicas dos profissionais: gênero, faixa etária e tempo de formado.

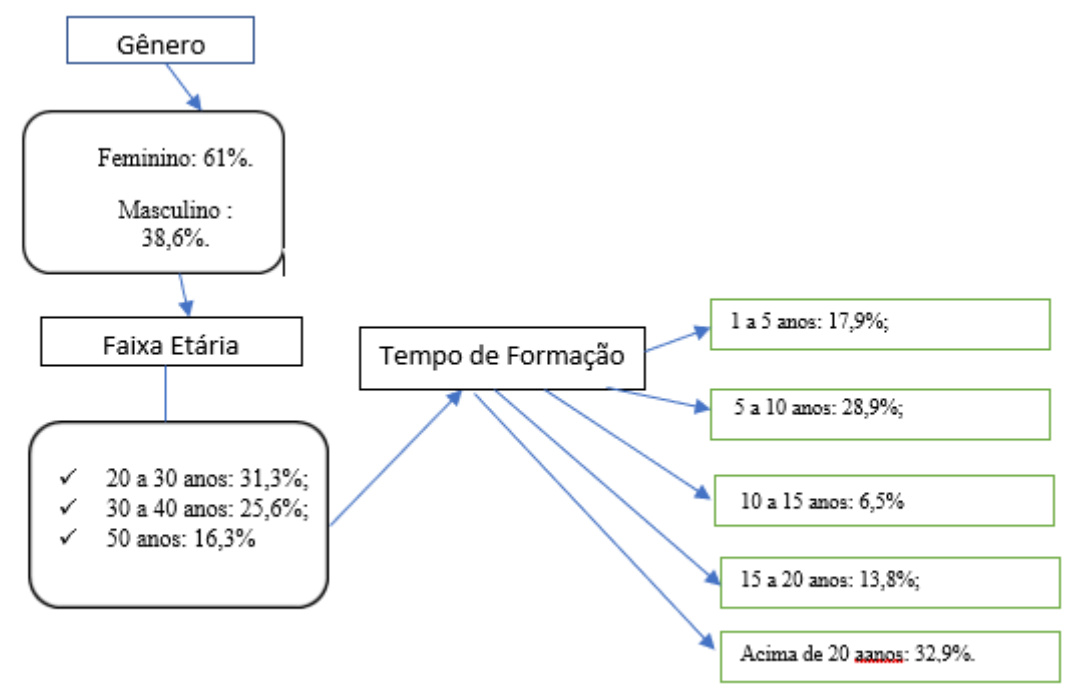

Fonte: Autores (2020). 
Figura 2 - Porcentagem do impacto econômico na rotina dos atendimentos odontológicos nas regiões Brasileiras.

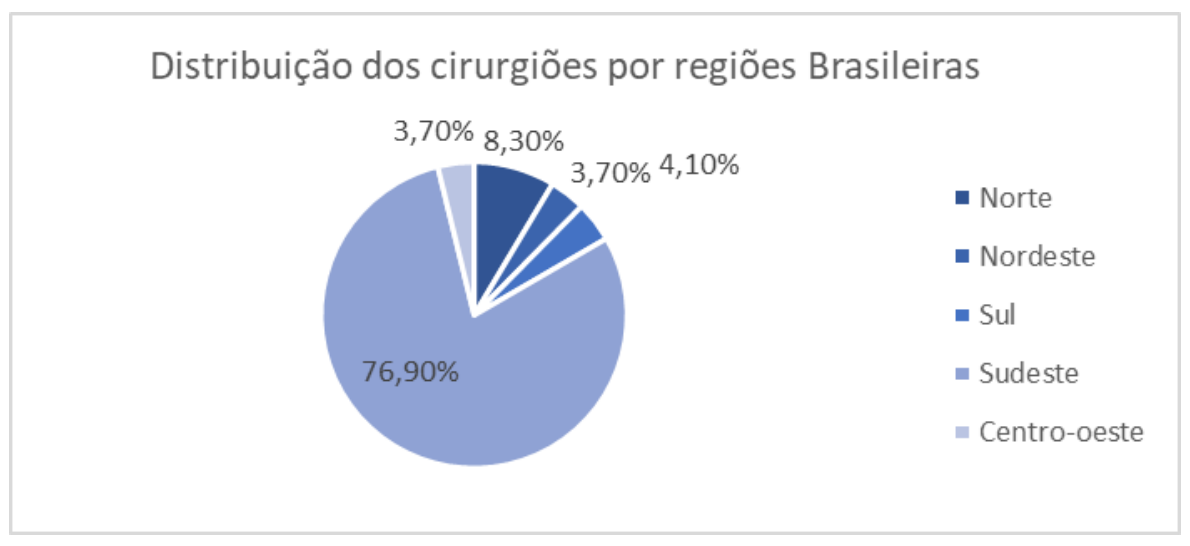

Fonte: Autores (2020).

Neste contexto, a OMS (2015) relata os efeitos negativos imediatos provocados pela pandemia e como consequência o cenário microeconômico assimétrico conforme a fragilidade da região, em relação ao tempo de permanência necessário de medidas de contenção do COVID-19, findando a desaceleração, corroborando com a figura acima.

A pandemia gerou um quadro caótico com elevado número de contaminados e mortos, o Brasil adotou a quarentena ou o auto isolamento como medida de contenção do vírus (OMS, 2020). Essa queda no fluxo de atendimento deve-se ao profissional realizar atendimentos específicos: urgência e emergência em um primeiro momento, após a OMS (2020) constatar como vetor de transmissão direta pelo COVID-19. A conscientização e divulgação em massa das informações sobre a infecção, demonstra que $42,3 \%$ dos profissionais relataram reciclagem do ministério da saúde para o enfrentamento da Pandemia do COVID-19 (Figura 3).

Figura 3 - Porcentagem dos cirurgiões dentistas que relataram uma abordagem educativo do ministério da Saúde para o enfrentamento da Pandemia do COVID-19.

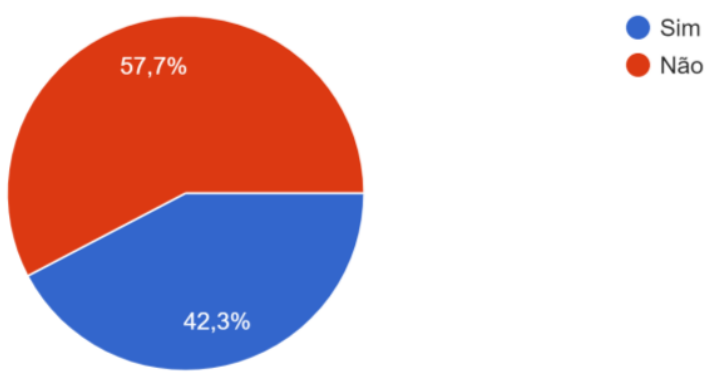

Fonte: Autores (2020).

Por conseguinte, o estudo revelou que a totalidade dos cirurgiões dentistas (246) promoveram conhecimento dos Protocolos para o atendimento odontológico no período do COVID-19, através de: site ministério da Saúde (37\%), site do conselho de Odontologia $(27,6 \%)$, colegas $(21,5 \%)$ e $(13,5 \%)$ revistas científicas (Figura 4). 
Figura 4 - Porcentagem dos diversos Métodos de busca pelos cirurgiões dentistas para conhecimento do Protocolo Odontológico do COVID-19.

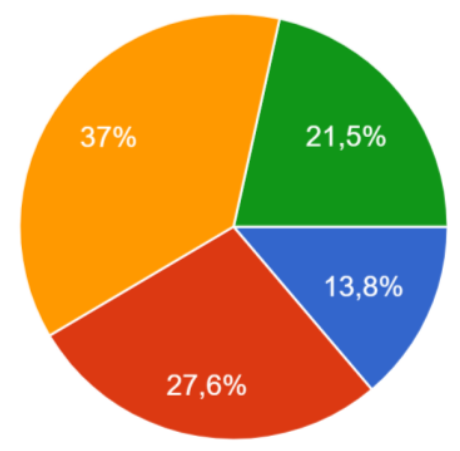

Colegas

Fonte: Autores (2020).

A crise provocou profunda reflexões na classe odontológica, diante da exposição e risco os cirurgiões dentistas, estes adotaram medidas protetivas e preventivas em relação a continuidade dos negócios e o andamento da economia de maneira que $(41,8 \%)$ investiram em Equipamento de proteção individual (Epi) seja por meio de loja virtual (38,9 \% ),seguidos de 22,5 $\%$ de lojas físicas, 3,3\% com conhecidos e 35,2\% relataram recorrer as lojas: físicas e virtuais de forma preventiva e protetiva ao COVID-19. A aquisição dos EPIS, acabou por ser um investimento variável, relatado pelos profissionais, variando entre: 100 a 500 reais $(41,8 \%),(32,2 \%)$ entre 500 a 1000 reais, $(11,7 \%$ ) entre 1000 a 1500 reais, $(9,2 \%)$ acima de 2000 e $5 \%$ relataram gastos entre 1500 a 2000 (Figura 5 ).

Figura 5 - Porcentagem do investimento dos cirurgiões dentistas na aquisição de Equipamentos de proteção individual, frente a Pandemia do COVID-19.

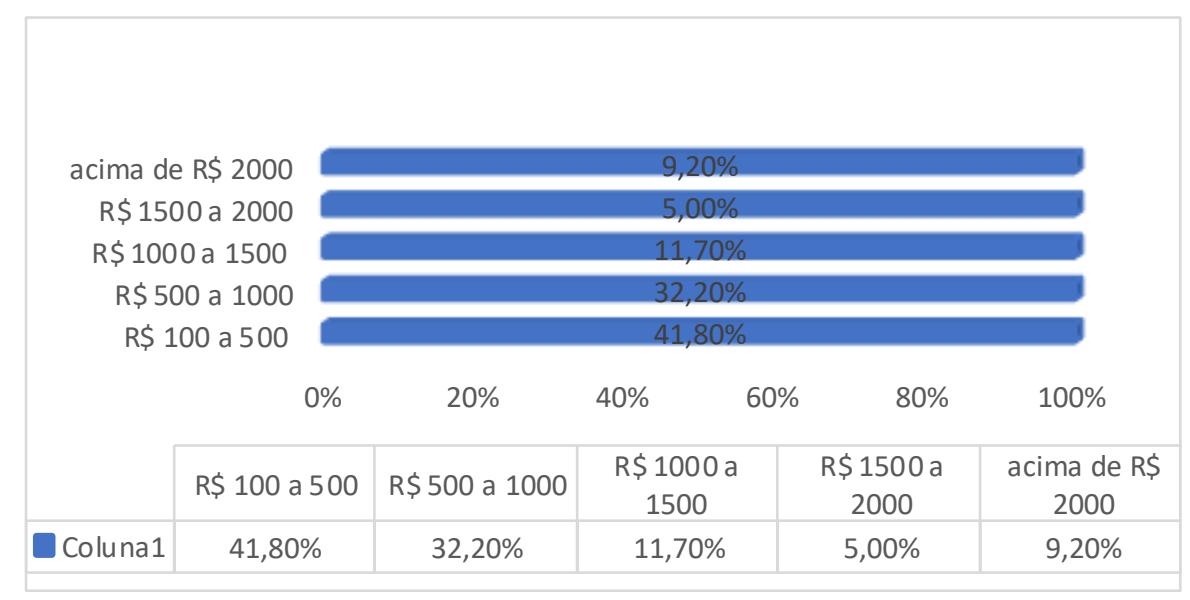

Fonte: Autores (2020).

Nos quesitos relacionados aos atendimentos realizados durante a pandemia, o atendimento emergencial foi o mais relatado com 44,3\%, seguido pelo atendimento clínico (30,9\%), exodontia (11\%), Ortodôntico (8,1\%), raspagem (0,8 \%), e 4,9 \% não relataram atendimento (Figura 6). 
Figura 6 - Porcentagem dos atendimentos realizados cirurgiões dentistas durante a Pandemia do COVID-19.

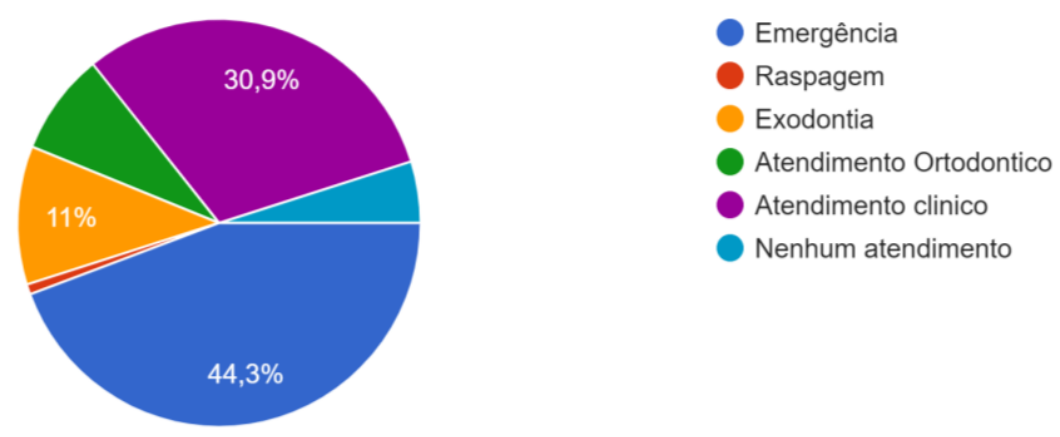

Fonte: Autores (2020).

Os cirurgiões dentistas durante a pandemia do COVID-19, estabeleceram as rotinas de trabalho de forma a estabelecer novas formas de atendimento odontológico aos clientes, destacando prioridades após o início da pandemia, como observado da figura 6.

O novo coronavírus trouxe sérios riscos à saúde dos profissionais de saúde, principalmente aos cirurgiões dentistas, o que permite realizar o levantamento e discussões em nosso estudo sobre a contaminação desses profissionais.

A contaminação provocou o afastamento desses profissionais de sua rotina de trabalho por: 01 a 10 dias $(17,5 \%)$, de 10 a 20 dias $(23,3 \%)$, de 20 a 30 dias (18,8\%), de 30 a 40 dias (12,1\%), e acima de 40 dias (28,3\%), o que talvez explique o agravamento de ordem sistêmica pelo COVID- 19 (Figura: 7).

Figura 7 - Porcentagem de cirurgiões dentistas contaminados pelo COVID-19, relacionados aos dias de afastamento do trabalho.

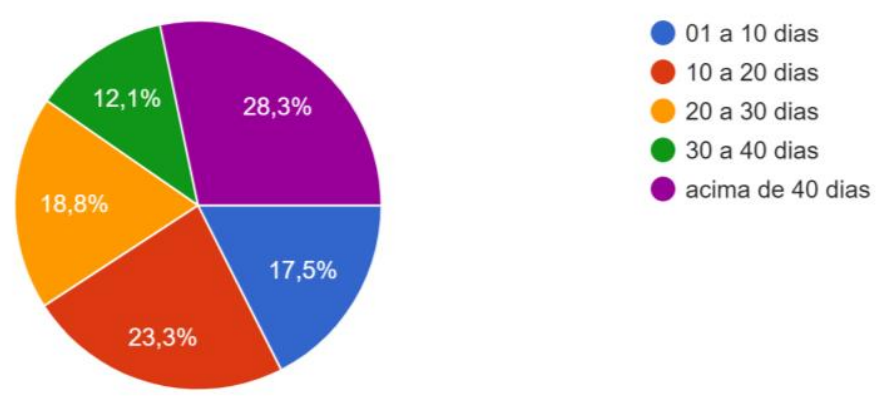

Fonte: Autores (2020).

Essa contaminação pode ter ocorrido por descuido desses 57,7\% (Figura 2), que não buscaram conhecimento através de curso para o enfrentamento da pandemia do COVID-19, contaminando se com maior facilidade, seja pela não utilização de produtos específicos para a desinfecção de superfície $(8,5 \%)$ ou os 3,3\% (8) que não utilizaram os equipamentos de proteção individual (Figura8). 
Figura 8 - Porcentagem do uso de EPIS e produtos de desinfecção de superfície durante a rotina de atendimentos odontológicos.

\begin{tabular}{|l|l|l|}
\hline & Sim & Não \\
\hline EPIS & $96,9 \%$ & \\
\hline $\begin{array}{l}\text { Produtos de desinfecção de } \\
\text { superfície }\end{array}$ & $8 \%$ & \\
\hline
\end{tabular}

Fonte: Autores (2020).

Os aspectos positivos da avaliação mencionados pelos cirurgiões dentistas foram a possibilidade de identificar os erros e dificuldades, gerados pela COVID-19, a fim de buscar alternativas para superá-los, e a motivação gerada por resultados satisfatórios.

\section{Considerações Finais}

Este artigo teve por objetivo avaliar o impacto econômico e mental da Pandemia do COVID-19 nas rotinas odontológicas dos cirurgiões dentistas no Brasil. A pesquisa buscou analisar as percepções e experiências relatadas pelos cirurgiões dentistas ocorridos durante a pandemia do COVID-19 e de que forma esses profissionais adquiriram conhecimento e aprendizado sobre a pandemia. As percepções emoções mais relatadas pelos cirurgiões dentistas foram: medo, irritabilidade, ansiedade, desespero, desmotivação, taquicardia, impaciência, pânico, frustração, estresse, síndrome de Burnout, insegurança, medo de contaminar-se, depressão, insônia, tristeza, cefaleia, intolerante, transtorno de humor, fobia. Neste contexto, de conhecimento limitado e restrito do vírus, provoca mudanças abruptas nas rotinas odontológicas, relacionadas a realização de uma entrevista e anamnese prévia ao atendimento, seja via telefônica ou online; associadas as restrições das especialidades de urgência e emergência.

As principais evidências do estudo, os cirurgiões dentistas enquanto promotores de saúde bucal convivem com o risco biológico, evidente ao número de profissionais afastados do exercício de sua profissão, devido a contaminação pelo COVID19. Embora, as sensações negativas estejam presentes, a minoria dos cirurgiões dentistas procura-se capacitar para o enfrentamento da pandemia, seja por meios formais ou informais.

O tema tem relevância; pois os resultados obtidos indicam a necessidade da reciclagem, considerando as influências dos problemas de saúde da COVID-19, sobre os processos de ensino e aprendizagem dos cirurgiões dentistas que estão inseridos no mercado de trabalho, sejam no âmbito público ou privado. Espera-se que o tema promova uma reflexão norteadora entre acadêmicos, profissionais, e poder público de modo a subsidiar possíveis repercussões sobre os cirurgiões dentistas.

Os cirurgiões dentistas enquanto profissionais sejam de entidades brasileiras: públicas ou privadas deveriam ter uma proposta de âmbito nacional de programa de educação financeira e psicológica durante a pandemia do COVID- 19; pois eles tiveram suas rotinas profissionais alteradas, com prejuízos econômicos, e expostos a riscos gerando alterações psicossociais.

\section{Referências}

Ahmed, M. Z., et al. (2020). Epidemic of COVID-19 in China and Associated Psychological Problems. Asian J Psych.

Banco Mundial (2020). Pandemia de Covid-19: choques na educação e respostas de políticas. http://pubdocs.worldbank.org/en/321431590757092620//Covid19-Education-Summary-port.pdf.

Banerjee, D. (2020).The COVID-19 outbreak: Crucial role the psychiatrists can play. Asian J Psych.

Brasil. (2020). Ministério da Saúde (MS). Brasil confirma primeiro caso da doença. https://coronavirus.saude.gov.br. 
Research, Society and Development, v. 10, n. 1, e22310111207, 2021 (CC BY 4.0) | ISSN 2525-3409 | DOI: http://dx.doi.org/10.33448/rsd-v10i1.11207

Brasil.(2020). Ministério da Saúde (MS). Corona vírus COVID-19. https://coronavirus.saude.gov.br/.

Brasil.(2020). Ministério da Saúde (MS). Painel coronavírus.

Holmes, E. A., et al (2020). Multidisciplinary research priorities for the COVID-19 pandemic: a call for action for mental health science. Lancet Psych.7(6):547-560

Huang, C., et al.(2020). Características clínicas de pacientes infectados com o novo coronavírus de 2019 em Wuhan, China. Lancet. 1(395): 497-506.

Morens, D. M., Folkers, G. K., \& Fauci, A. S. (2009). What is a pandemic? J Infect Dis. 200(7):1018-1021.

Pan American Health Organization. (2020). Folha informativa COVID-19. http://www.paho.org/index.php?option=com_content\&viem=a rticle\&id=6101: covid19\&Itemid=875\#datas-notificacoes .

Peng, X. et al.(2020). Rotas de transmissão de controles nCoVand 2019na prática odontológica. Int J Oral Sci. 1(12), 9.

WHO. (2020).Coronavirus disease 2019 (COVID-19): situation report. 